\title{
1. Extending the Hydrophobic Mismatch Concept to Amphiphilic Membranolytic Peptides
}

\author{
3 Ariadna Grau-Campistany, ${ }^{\ddagger \dagger}$ Erik Strandberg, ${ }^{\ddagger}$ Parvesh Wadhwani, ${ }^{\ddagger}$ Francesc Rabanal, \\ 4 and Anne S. Ulrich**, ${ }^{*}, \S$ \\ 5 Institute of Biological Interfaces (IBG-2), Karlsruhe Institute of Technology (KIT), POB 3640, 76021 Karlsruhe, Germany \\ $6{ }^{\dagger}$ Departament de Química Orgànica, Facultat de Química, Universitat de Barcelona, Barcelona, Spain \\ $7{ }^{\S}$ Institute of Organic Chemistry, KIT, Fritz-Haber-Weg 6, 76131 Karlsruhe, Germany
}

8 S Supporting Information

9 ABSTRACT: A series of nine amphiphilic, pore-forming $\alpha$-helical KIA peptides

10 (KIAGKIA repeats) with lengths between 14 and 28 residues were studied by solid-

11 state ${ }^{15} \mathrm{~N}$ NMR to determine their alignment in oriented lipid bilayers. In a 2:1 mixture of

12 1,2-dimyristoyl-sn-glycero-3-phosphatidylglycerol (DMPC) with its corresponding 1-

13 myristoyl-2-hydroxy-sn-glycero-3-phosphocholine (lyso-MPC), which has a highly positive

14 spontaneous curvature, the helix tilt angle was found to vary steadily with peptide length.

15 The shortest peptide was aligned transmembrane and upright, while the longer ones

16 successively became tilted away from the membrane normal. This behavior is in agreement

17 with the hydrophobic matching concept, conceived so far only for hydrophobic helices. In

18 1,2-dioleoyl-sn-glycero-3-phosphatidylcholine, with a negative spontaneous curvature, all KIA peptides remained flat on the

19 bilayer surface, while the cylindrical DMPC lipids permitted a slight tilt. Peptide insertion thus depends critically on the intrinsic

lipid curvature, and helix orientation is then fine-tuned by membrane thickness. A refined toroidal pore model is proposed.

21 Tydrophobic matching is a well-known concept that has 22 been widely applied to hydrophobic transmembrane 23 segments of proteins. However, its potential relevance for 24 amphiphilic helices has been largely ignored, because many 25 other parameters limit the ability of an amphiphilic peptide to 26 insert into a lipid bilayer in the first place. These include the 27 peptide-to-lipid ratio, ${ }^{2-5}$ temperature, ${ }^{6}$ sample hydration, ${ }^{7} \mathrm{pH},{ }^{8}$ 28 lipid composition, ${ }^{9-11}$ as well as peptide amphiphilicity and 29 interfacial activity. ${ }^{12}$ Among the various physical properties of 30 the bilayer, the spontaneous curvature of the lipids has been 31 recently suggested to play a pivotal role in allowing peptides to 32 flip from a surface-bound to a tilted or inserted transmembrane 33 state. $^{9-11}$ Here, we have utilized bilayers with a highly positive 34 spontaneous curvature to promote the ability of amphiphilic 35 peptides to insert and assemble as stable toroidal pores. These 36 data prove that (i) the intrinsic lipid curvature is a critical 37 parameter that allows membrane insertion to occur (rather than 38 membrane thickness) and (ii) once inserted, the helix tilt angle 39 is governed by hydrophobic mismatch, as had been described 40 only for hydrophobic segments so far. ${ }^{13,14}$ Bilayer thickness 41 thus determines only the "fine-tuning" of the helix tilt angle, 42 and based on these findings, a new and refined model of a 43 toroidal pore can be presented.

44 A series of nine ideally amphiphilic $\alpha$-helical peptides, called 45 KIA peptides (whose sequences consist of KIAGKIA repeats, 46 see Table 1 ), were synthesized with lengths between 14 and 28 47 amino acids and were shown to be highly helical when bound 48 to membranes (Table 1). ${ }^{15}$ We had previously found that these 49 peptides show antimicrobial and hemolytic activity and can 50 induce leakage in small unilamellar vesicles depending on peptide length. Leakage is induced only when the length of the 51 peptide is at least as long as the hydrophobic thickness of the 52 membrane, indicating that the mechanism of action invokes 53 genuine pore formation with peptides spanning the membrane, 54 with the peptide axis parallel to the membrane normal. ${ }^{15} \quad 55$

The orientations of the KIA peptides in membranes were 56 determined by solid-state ${ }^{15} \mathrm{~N}$ NMR in aligned samples, as 57 previously described. ${ }^{15}$ The helix tilt angle was estimated from 58 the chemical shift of a ${ }^{15} \mathrm{~N}$-Ala label at position 10 in the 59 backbone, ${ }^{9,16}$ and ${ }^{31} \mathrm{P}$ NMR was used to monitor any 60 perturbations of the phospholipid bilayers. The NMR experi- 61 ments were performed in different lipid systems with varying 62 spontaneous curvature, using typically $1 \mathrm{mg}$ of labeled peptide 63 per sample. Considering the different lengths of the KIA 64 peptides, their concentrations were chosen such that the 65 number of amino acid residues per lipid was kept constant, with 66 a peptide-to-lipid molar ratio $(\mathrm{P} / \mathrm{L})$ of $1: 50$ for KIA21 as a 67 reference point. When bound to the membrane surface, all 68 peptides should thus cover a comparable area and cause a 69 similar degree of membrane perturbation.

70

The NMR spectra in Figure 1A show that all peptides gave a $71 \mathrm{fl}$ peak close to $90 \mathrm{ppm}$ in 1,2-dioleoyl-sn-glycero-3-phosphati- 72 dylcholine (DOPC), indicating a flat, surface-bound state. In a 73 mixture of 1-palmitoyl-2-oleoyl-sn-glycero-3-phosphatidylcho- 74 line (POPC) with the anionic 1-palmitoyl-2-oleoyl-sn-glycero- 75

Received: January 21, 2016

Accepted: February 25, 2016 
Table 1. Peptides Used in This Study ${ }^{a}$

\begin{tabular}{|c|c|c|c|}
\hline peptide & sequence & helicity $(\%)^{b}$ & $\mathrm{P} / \mathrm{L}^{c}$ \\
\hline KIA14 & KIAGKIA KIAGGIA-NH ${ }_{2}$ & 74.1 & $1: 34$ \\
\hline KIA15 & KIAGKIA KIAGKIA K-NH ${ }_{2}$ & 68.7 & $1: 37$ \\
\hline KIA17 & KIAGKIA KIAGKIA KIA-NH ${ }_{2}$ & 82.7 & $1: 41$ \\
\hline KIA19 & KIAGKIA KIAGGIA KIAGK-NH ${ }_{2}$ & 81.7 & $1: 46$ \\
\hline KIA21 & KIAGKIA KIAGGKIA KIAGKIA-NH ${ }_{2}$ & 83.0 & $1: 50$ \\
\hline KIA22 & KIAGKIA KIAGGKIA KIAGKIA K-NH ${ }_{2}$ & 67.7 & $1: 54$ \\
\hline KIA24 & KIAGKIA KIAGKIA KIAGKIA KIA-NH & 72.6 & $1: 58$ \\
\hline KIA26 & KIAGKIA KIAGGKIA KIAGKIA KIAGK-NH ${ }_{2}$ & 71.1 & $1: 62$ \\
\hline KIA28 & KIAGKIA KIAGGKIA KIAGKIA KIAGKIA-NH & 76.2 & $1: 66$ \\
\hline
\end{tabular}

${ }^{a_{\text {The }}}$ underlined Ala-10 was labeled with ${ }^{15} \mathrm{~N}$ in the backbone amide. ${ }^{b}$ Percentage $\alpha$-helix according to a deconvolution of circular dichroism spectra. ${ }^{15}{ }^{c}$ Peptide-to-lipid molar ratio in samples with a constant peptide-to-lipid mass ratio.

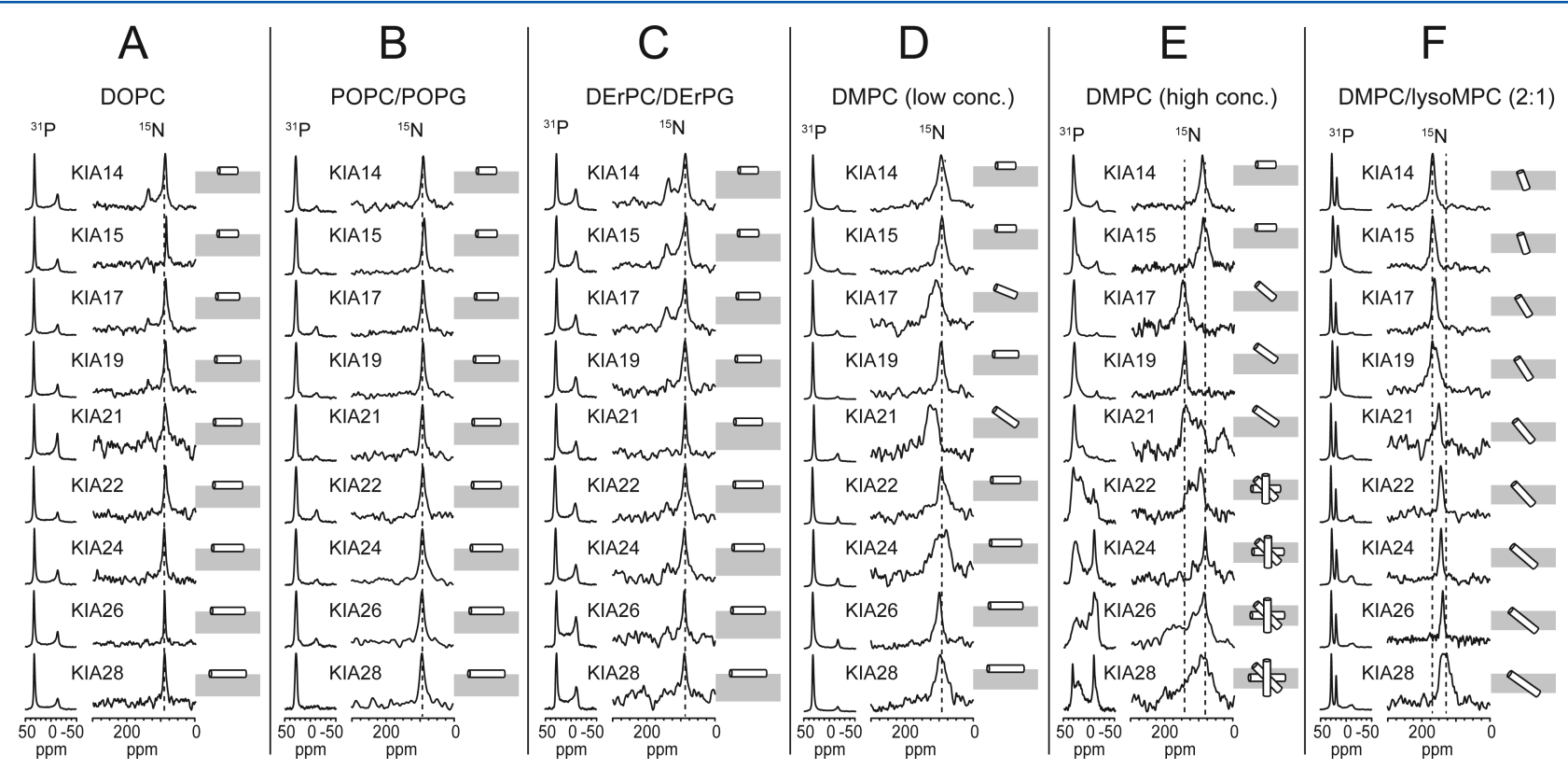

Figure 1. Solid-state ${ }^{31} \mathrm{P}$ and ${ }^{15} \mathrm{~N}$ NMR of KIA peptides with varying lengths, measured in oriented samples composed of lipids with different spontaneous curvatures. (A) DOPC (20 mg, $1.2 \mathrm{mg}$ peptide); (B) POPC/POPG (6.5 mg each, $0.8 \mathrm{mg}$ peptide); (C) DErPC/DErPG (10 mg each, $1.1 \mathrm{mg}$ peptide); (D) DMPC (15 mg, $1.1 \mathrm{mg}$ peptide); (E) DMPC at a high P/L ratio of 1:20; (F) DMPC/lyso-MPC (2:1) (12 mg DMPC, $5 \mathrm{mg}$ lyso-MPC, $1.2 \mathrm{mg}$ peptide). Except for column E, the peptide-to-lipid mass ratio was kept constant (and corresponds to a commonly used molar $\mathrm{P} / \mathrm{L}$ ratio of 1:50 for the medium-length KIA21). Vertical dotted lines are included in the spectra to guide the eye, and the estimated peptide orientation is illustrated for each case.

76 3-phosphatidylglycerol (POPG) (1:1), the same behavior was 77 found (Figure 1B). It has been suggested that negative 78 spontaneous curvature (i.e., voluminous acyl chains with 79 relatively small head groups) is unfavorable for peptide 80 insertion. ${ }^{10}$ Hence, it is not surprising that none of the KIA 81 peptides would insert into these lipids. ${ }^{17}$ Also in 1,2-dierucoyl82 sn-glycero-3-phosphatidylcholine (DErPC)/1,2-dierucoyl-sn83 glycero-3-phosphatidylglycerol (DErPG) (1:1), with long 84 unsaturated chains (22 carbon atoms), no insertion was 85 observed. All these lipid systems support our previous findings 86 that amphiphilic peptides (in which multiple cationic residues 87 evenly distributed along the length of the helix) generally 88 remain surface-bound in unsaturated PC bilayers. ${ }^{9} 10,18$

89 In 1,2-dimyristoyl-sn-glycero-3-phosphatidylglycerol 90 (DMPC), a lipid with a small positive spontaneous curvature, 91 the KIA peptides were also found to lie more or less flat on the 92 membrane surface (Figure 1D). Only a slight insertion was 93 expected, and indeed the peaks of KIA17 and KIA21 are seen 94 to be shifted to $\sim 120 \mathrm{ppm}$. Samples were therefore also 95 prepared with a higher $\mathrm{P} / \mathrm{L}$ of 1:20 to promote overall insertion. This set of data shows a clear dependence on peptide 96 length, as seen in Figure 1E. For KIA14 and KIA15, the signal 97 at $90 \mathrm{ppm}$ indicates a surface orientation, while KIA17 and 98 KIA19 with a chemical shift around $150 \mathrm{ppm}$ exhibit a more 99 inserted state. KIA21 and KIA22 gave broad line shapes, where 100 the signal at $150 \mathrm{ppm}$ represents inserted peptides, while the 90101 ppm peak arises from large unoriented aggregates that give rise 102 to a powder spectrum with a $90 \mathrm{ppm}$ maximum. KIA24 also has 103 a maximum around 90 ppm, yet both KIA24 and KIA26 show 104 very poor orientation in the membrane, as seen from the ${ }^{31} \mathrm{P} 105$ spectra (Figure 1E). KIA26 and KIA28, finally, gave mostly 106 powder spectra in both ${ }^{31} \mathrm{P}$ and ${ }^{15} \mathrm{~N}$ NMR , indicating that 107 these peptides at such high $\mathrm{P} / \mathrm{L}$ ratio formed immobilized 108 aggregates, which perturbed the membranes and destroyed the 109 bilayer orientation.

After comparing these conventional lipids, which do not tend 111 to readily support peptide insertion, we prepared another series 112 of samples with DMPC and its corresponding lyso-lipid (1- 113 myristoyl-2-hydroxy-sn-glycero-3-phosphocholine, lyso-MPC), 114 which has a very high positive spontaneous curvature, at a 115 
116 2:1 molar ratio. This optimized lipid mixture facilitated 117 insertion of the KIA peptides remarkably well, even at low 118 concentration (using the same mass of peptide in each sample, 119 as above). Under these conditions, we observed for the first 120 time an exemplary length-dependent realignment of amphi121 philic peptides. Namely, a distinct, gradual change in the ${ }^{15} \mathrm{~N}$ 122 chemical shift is seen in Figure 1F, without any indication of 123 problematic peptide aggregation or membrane perturbation, as 124 confirmed by ${ }^{31} \mathrm{P}$ NMR. The shortest peptide, KIA14, gave a 125 signal at $167 \mathrm{ppm}$, indicating a fully inserted transmembrane 126 state in which the helix axis is essentially parallel to the 127 membrane normal (much more upright than what was seen in 128 DMPC, Figure 1E). The longer peptides then showed gradually 129 smaller chemical shifts down to 134 ppm for KIA28. This series 130 indicates that the shortest peptides are almost fully upright in 131 the membrane, while the longer ones exhibit a steady decrease 132 in tilt angle, until the longest ones end up in an obliquely tilted 133 state. The latter scenario resembles that of KIA21 (also called 134 MSI-103) in DMPC at $\mathrm{P} / \mathrm{L}=1: 50$, where a tilt angle of $125^{\circ}$ 135 was previously found by ${ }^{2} \mathrm{H}$ NMR., 90,19

136 The same type of tilting response has been reported and 137 extensively discussed for completely hydrophobic, designated 138 transmembrane segments. ${ }^{13,14}$ The new data suggest that also 139 for amphiphilic peptides a larger tilt angle seems to be 140 necessary for the longer helices to avoid hydrophobic 141 mismatch. A more detailed evaluation of the quantitative 142 relationship between chemical shift and peptide orientation, 143 supporting the conclusions and illustrations drawn here, can be 144 found in the Supporting Information. We briefly note that our 145 samples were also measured at a vertical alignment, showing 146 that the peptides rotated rapidly around the membrane normal; 147 this was the case for both long and short peptides in all lipid 148 systems (see Figure S1 in the Supporting Information). Such 149 behavior had been previously observed for surface-bound and 150 tilted amphiphilic peptides ${ }^{9,10,20}$ but was unknown so far for 151 their oligomeric pore-forming transmembrane states. A fast 152 rotation around the membrane normal has also been observed 153 for other types of membrane-spanning peptides in liquid 154 crystalline lipid bilayers. ${ }^{21-23}$

155 Interestingly, in DMPC the abrupt change in chemical shift 156 upon going from KIA15 (90 ppm) to KIA17 (150 ppm) 157 indicates that the peptides can get inserted only provided that 158 they are long enough to span the membrane. This observation 159 suggests that membrane lysis by these amphiphilic peptides 160 invokes the formation of genuine oligomeric transmembrane 161 pores. $^{15}$ In a recent study, the orientations of KIA peptides 162 found from ${ }^{15} \mathrm{~N}$ NMR did not correlate directly with membrane 163 leakage in unsaturated lipids, ${ }^{15}$ but they could nevertheless be 164 reconciled with activity. Leakage occurs only when pores are 165 formed, but when a small population of peptides is assembled 166 as transient, short-lived pores, this is already sufficient to cause 167 considerable leakage. On the other hand, ${ }^{15} \mathrm{~N}$ NMR provides a 168 steady-state, time-averaged picture of all peptides, and if only a 169 small fraction exists in the transmembrane state at any given 170 time, these pores are hardly visible in the NMR spectra. Here, 171 the unconventional DMPC/lyso-MPC lipid mixture allowed 172 the entire peptide population to insert into the membrane and 173 to evidently assemble as stable toroidal pores, which could be 174 conveniently studied using solid-state NMR. Under these 175 conditions, the $\alpha$-helical KIA peptides were found to readily 176 adjust their tilt angle according to their respective lengths, in 177 full agreement with the concept of hydrophobic matching. This 178 study demonstrates that the straightforward biophysical description of hydrophobic mismatch not only applies to 179 transmembrane protein segments but also can now be extended 180 toward amphiphilic peptides.

The pores formed by KIA peptides are most likely of the 182 toroidal type which has been proposed for similar amphipathic 183 peptides like magainin $2,{ }^{24,25}$ with the hydrophilic face of the 184 peptides lining the pore, and lipid head groups being 185 interspersed between the peptides (Figure 2). As discussed in $186 \mathrm{f} 2$
A
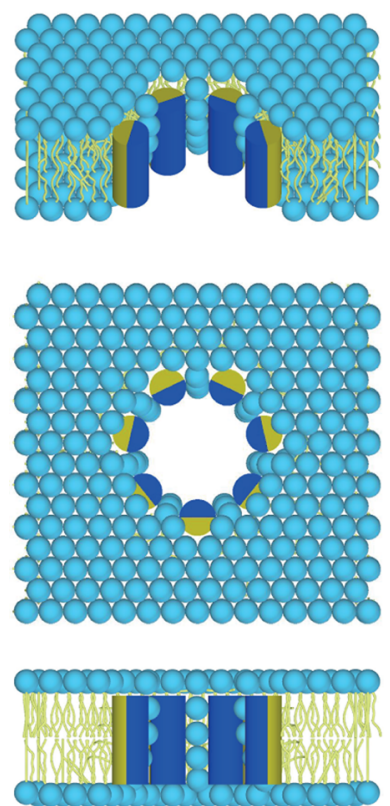
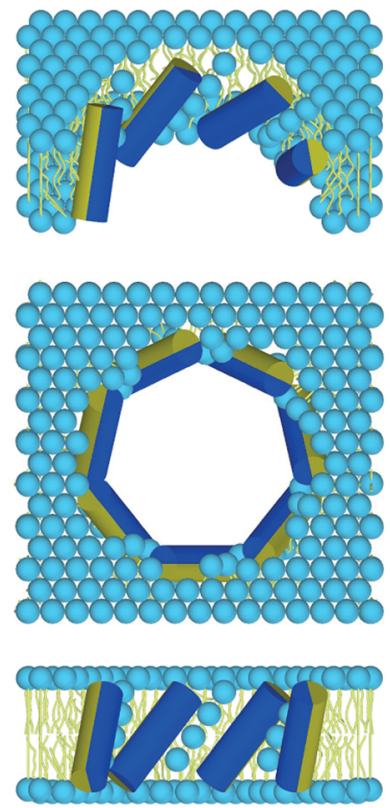

Figure 2. Model of a transmembrane toroidal pore formed by KIA peptides. The lipids are represented with light blue head groups and yellow acyl chains. The peptides are shown as cylinders with one hydrophobic face (dark blue) and one polar, hydrophilic face (gold). The inside of the pore is lined by the polar faces of the peptides and presumably also by lipid head groups that are intercalated between the helices. (A) Short peptides matching the hydrophobic thickness of the membrane have a straight upright orientation. (B) Longer peptides adapt to the membrane thickness by tilting, forming an iris-like ring of helices lining the pore, presumably together with some lipid head groups.

the Supporting Information, the ${ }^{31} \mathrm{P}$ NMR spectra are 187 compatible with such pores (see Figure 1, and Figure S2). 188 The mean curvature in the interior of a toroidal pore can be 189 rather small, corresponding to a saddle with a negative Gaussian 190 modulus. It has nevertheless been suggested that lipids with a 191 positive spontaneous curvature, such as the lyso-lipids used 192 here, are preferentially localized in these regions, facilitating and 193 stabilizing toroidal pores. $^{26-28}$ It was originally suggested that 194 negatively charged lipid head groups are necessary inside the 195 pore to neutralize the positive charges of the peptides and to 196 counteract their electrostatic repulsion, ${ }^{25}$ but recent results 197 indicate that this is not the case. For example, in pure PC lipid 198 systems, we have observed the cationic amphiphilic antimicro- 199 bial peptide PGLa in a distinct transmembrane orientation, 200 assembled with magainin 2 into a pore-forming complex, ${ }^{9}$ and 201 here we also find KIA peptide pores in PC/lyso-PC lipids, 202 where no negatively charged lipids are present. A recent 203 molecular dynamics simulation also indicated that pores of 204 
205 melittin were more stable in DMPC than in DMPC/DMPG 206 bilayer, in line with experimental results. ${ }^{29}$

207 The idea of hydrophobic mismatch was conceived for 208 "classical" transmembrane segments of integral membrane 209 proteins, i.e., when the helices are completely hydrophobic 210 (and obviously have no pore-forming function). This concept 211 was found to apply ideally when a single hydrophobic peptide 212 crosses the membrane, while helix bundles in polytopic 213 membrane proteins tend to interact with one another to 214 stabilize their individual tilt angles. ${ }^{13,30}$ In the case of the 215 amphiphilic pore-forming peptides, however, a soft oligomeric 216 assembly has to be considered, where the individual 217 components can readily slide along each other like the 218 segments of an iris, "greased" by the intercalated lipid head 219 groups.

220 Depending on the global or local point of view, the (global) 221 "transmembrane" alignment of the KIA peptides within the 222 pore is fully consistent with the statement that the peptides are 223 at the same time (locally) floating on the amphiphilic "surface" 224 of the lipid monolayer lining the toroidal wormhole. If the 225 peptides were free to diffuse within the continuous monolayer 226 leaflet, one might expect that the helices could assume any tilt 227 angle simply by shifting their position along the surface within 228 the toroidal pore. However, this is not observed by NMR, 229 because there is not a broad distribution of NMR signals. 230 Instead, a single sharp peak (Figure $1 F$ ) with a characteristic 231 chemical shift is observed, indicating that all peptides have very 232 similar orientations and that they do not undergo averaging by 233 rapid passages across the toroidal pore. It thus seems there are 234 two stable orientations for KIA peptides in lipid bilayers: either 235 flat on the (global) membrane surface or more or less tilted 236 within the center of the pore, but nothing in between. This 237 would fit with a "classical" toroidal pore as illustrated for 238 example by Brogden. ${ }^{31}$ Under the equilibrium conditions of the 239 NMR experiment, we conclude that the ideally amphiphilic $\alpha$ 240 helical KIA peptides are able to assemble into a stable pore 241 complex, containing an unknown number of helices, as shown 242 in Figure 2. When the peptides are just long enough to span the 243 bilayer, they are aligned fully upright with the helix axis parallel 244 to the membrane normal (Figure 2A). When the peptides are 245 longer (Figure 2B), they will tilt within the complex like an iris, 246 such that the hydrophobic residues (e.g., Ile-2) are still retained 247 within the hydrophobic region of the bilayer. This refined 248 toroidal pore model can account for the length-dependent 249 differences in helix tilt angles, observed here for the first time in 250 a suitable lipid matrix. In these pores, peptides have a global 251 transmembrane orientation along the membrane normal and 252 their tilt angle depends on the length of the helix and the 253 hydrophobic thickness of the bilayer, in a similar way as the tilt 254 of hydrophobic helices is known to depend on hydrophobic 255 matching with the membrane. This mismatch dependence of 256 amphiphilic helical peptides had not been observed previously. 257 By using lipids with a high positive spontaneous curvature, 258 we have shown that it is possible to stabilize transmembrane 259 pores made up of amphiphilic peptides. In most other lipid 260 systems and under biological conditions, such pore structures 261 should be just as plausible, but are obviously only short-lived. 262 These transient events are hard to study with biophysical 263 methods, unless lipids with a high positive spontaneous 264 curvature are included, such as the lyso-MPC in the present 265 case. The use of lipids with a high positive spontaneous 266 curvature had so far been explored only sporadically. ${ }^{18}$ The 267 present study implies that the simple idea of including lyso- lipids should be rather useful in future studies, where other 268 putative pore-forming amphiphilic peptides need to be 269 characterized and compared in a stable transmembrane state. 270

\section{ASSOCIATED CONTENT}

\section{S Supporting Information}

272

The Supporting Information is available free of charge on the 273 ACS Publications website at DOI: 10.1021/acs.jpclett.6b00136. 274

Experimental methods, a discussion of peptide orienta- 275 tions determined from ${ }^{15} \mathrm{~N}$ chemical shifts, data on 276 rotational diffusion of peptides, and an analysis of ${ }^{31} \mathrm{P} 277$ NMR data (PDF)

\section{AUTHOR INFORMATION}

*E-mail: anne.ulrich@kit.edu. Tel: +49-(0)721-608-23222. Fax: 281 +49-(0)721-608-24823.

Notes

The authors declare no competing financial interest.

\section{ACKNOWLEDGMENTS}

We thank Daniel Gauder and Simon Hertweck for preparing 286 Figure 2. We thank Markus Schmitt for help with the NMR 287 infrastructure. A.G.-C. acknowledges financial support from the 288 DAAD (Scholarship A/12/77249) and EMBO (Short-Term 289 Fellowship ASTF 530-2012).

\section{REFERENCES}

(1) Killian, J. A.; Nyholm, T. K. Peptides in lipid bilayers: the power 292 of simple models. Curr. Opin. Struct. Biol. 2006, 16, 473-479. 293

(2) Strandberg, E.; Wadhwani, P.; Tremouilhac, P.; Dürr, U. H. N.; 294 Ulrich, A. S. Solid-state NMR analysis of the PGLa peptide orientation 295 in DMPC bilayers: structural fidelity of ${ }^{2} \mathrm{H}$-labels versus high 296 sensitivity of ${ }^{19}$ F-NMR. Biophys. J. 2006, 90, 1676-1686.

297

(3) Tremouilhac, P.; Strandberg, E.; Wadhwani, P.; Ulrich, A. S. 298 Conditions affecting the re-alignment of the antimicrobial peptide 299 PGLa in membranes as monitored by solid state ${ }^{2} \mathrm{H}-\mathrm{NMR}$. Biochim. 300 Biophys. Acta, Biomembr. 2006, 1758, 1330-1342.

(4) Glaser, R. W.; Sachse, C.; Dürr, U. H. N.; Afonin, S.; Wadhwani, 302 P.; Strandberg, E.; Ulrich, A. S. Concentration-dependent realignment 303 of the antimicrobial peptide PGLa in lipid membranes observed by 304 solid-state ${ }^{19}$ F-NMR. Biophys. J. 2005, 88, 3392-3397.

(5) Wadhwani, P.; Bürck, J.; Strandberg, E.; Mink, C.; Afonin, S.; 306 Ulrich, A. S. Using a sterically restrictive amino acid as a ${ }^{19} \mathrm{~F}-\mathrm{NMR} 307$ label to monitor and control peptide aggregation in membranes. J. Am. 308 Chem. Soc. 2008, 130, 16515-16517.

(6) Afonin, S.; Grage, S. L.; Ieronimo, M.; Wadhwani, P.; Ulrich, A. S. 310 Temperature-dependent transmembrane insertion of the amphiphilic 311 peptide PGLa in lipid bilayers observed by solid state ${ }^{19} \mathrm{~F}-\mathrm{NMR} 312$ spectroscopy. J. Am. Chem. Soc. 2008, 130, 16512-16514.

(7) Yang, L.; Harroun, T. A.; Weiss, T. M.; Ding, L.; Huang, H. W. 314 Barrel-stave model or toroidal model? A case study on melittin pores. 315 Biophys. J. 2001, 81, 1475-1485.

(8) Misiewicz, J.; Afonin, S.; Ulrich, A. S. Control and role of $\mathrm{pH}$ in 317 peptide-lipid interactions in oriented membrane samples. Biochim. 318 Biophys. Acta, Biomembr. 2015, 1848, 833-841.

(9) Strandberg, E.; Zerweck, J.; Wadhwani, P.; Ulrich, A. S. 320 Synergistic insertion of antimicrobial magainin-family peptides in 321 membranes depends on the lipid spontaneous curvature. Biophys. J. 322 2013, 104, L9-11.

(10) Strandberg, E.; Tiltak, D.; Ehni, S.; Wadhwani, P.; Ulrich, A. S. 324 Lipid shape is a key factor for membrane interactions of amphipathic 325 helical peptides. Biochim. Biophys. Acta, Biomembr. 2012, 1818, 1764- 326 1776. 
328 (11) Afonin, S.; Glaser, R. W.; Sachse, C.; Salgado, J.; Wadhwani, P.; 329 Ulrich, A. S. ${ }^{19}$ F NMR screening of unrelated antimicrobial peptides 330 shows that membrane interactions are largely governed by lipids. 331 Biochim. Biophys. Acta, Biomembr. 2014, 1838, 2260-2268.

332 (12) Wimley, W. C. Describing the mechanism of antimicrobial 333 peptide action with the interfacial activity model. ACS Chem. Biol. 334 2010, 5, 905-917.

335 (13) Strandberg, E.; Esteban-Martín, S.; Ulrich, A. S.; Salgado, J. 336 Hydrophobic mismatch of mobile transmembrane helices: merging 337 theory and experiments. Biochim. Biophys. Acta, Biomembr. 2012, 1818, 338 1242-1249.

339 (14) Park, S. H.; Opella, S. J. Tilt angle of a trans-membrane helix is 340 determined by hydrophobic mismatch. J. Mol. Biol. 2005, 350, 310341318.

342 (15) Grau-Campistany, A.; Strandberg, E.; Wadhwani, P.; Reichert, 343 J.; Bürck, J.; Rabanal, F.; Ulrich, A. S. Hydrophobic mismatch 344 demonstrated for membranolytic peptides, and their use as molecular 345 rulers to measure bilayer thickness in native cells. Sci. Rep. 2015, 5, 3469388.

347 (16) Bechinger, B.; Gierasch, L. M.; Montal, M.; Zasloff, M.; Opella, $348 \mathrm{~S}$. J. Orientations of helical peptides in membrane bilayers by solid 349 state NMR spectroscopy. Solid State Nucl. Magn. Reson. 1996, 7, 185350191.

351 (17) Kollmitzer, B.; Heftberger, P.; Rappolt, M.; Pabst, G. Monolayer 352 spontaneous curvature of raft-forming membrane lipids. Soft Matter 353 2013, 9, 10877-10884.

354 (18) Strandberg, E.; Ulrich, A. S. AMPs and OMPs: Is the folding 355 and bilayer insertion of $\beta$-stranded outer membrane proteins governed 356 by the same biophysical principles as for $\alpha$-helical antimicrobial 357 peptides? Biochim. Biophys. Acta, Biomembr. 2015, 1848, 1944-1954. 358 (19) Strandberg, E.; Kanithasen, N.; Bürck, J.; Wadhwani, P.; Tiltak, 359 D.; Zwernemann, O.; Ulrich, A. S. Solid state NMR analysis comparing 360 the designer-made antibiotic MSI-103 with its parent peptide PGLa in 361 lipid bilayers. Biochemistry 2008, 47, 2601-2616.

362 (20) Wadhwani, P.; Strandberg, E.; van den Berg, J.; Mink, C.; Bürck, 363 J.; Ciriello, R.; Ulrich, A. S. Dynamical structure of the short 364 multifunctional peptide BP100 in membranes. Biochim. Biophys. Acta, 365 Biomembr. 2014, 1838, 940-949.

366 (21) Van der Wel, P. C. A.; Strandberg, E.; Killian, J. A.; Koeppe, R. 367 E., II. Geometry and intrinsic tilt of a tryptophan-anchored 368 transmembrane $\alpha$-helix determined by ${ }^{2} \mathrm{H}$ NMR. Biophys. J. 2002, $36983,1479-1488$.

370 (22) Smith, R.; Separovic, F.; Milne, T. J.; Whittaker, A.; Bennett, F. 371 M.; Cornell, B. A.; Makriyannis, A. Structure and orientation of the 372 pore-forming peptide, melittin, in lipid bilayers. J. Mol. Biol. 1994, 241, 373 456-466.

374 (23) Cornell, B. A.; Separovic, F.; Baldassi, A. J.; Smith, R. 375 Conformation and orientation of gramicidin a in oriented 376 phospholipid bilayers measured by solid state carbon-13 NMR. 377 Biophys. J. 1988, 53, 67-76.

378 (24) Hallock, K. J.; Lee, D. K.; Ramamoorthy, A. MSI-78, an 379 analogue of the magainin antimicrobial peptides, disrupts lipid bilayer 380 structure via positive curvature strain. Biophys. J. 2003, 84, 3052-3060. 381 (25) Cruciani, R. A.; Barker, J. L.; Durell, S. R.; Raghunathan, G.; 382 Guy, H. R.; Zasloff, M.; Stanley, E. F. Magainin 2, a natural antibiotic 383 from frog skin, forms ion channels in lipid bilayer membranes. Eur. J. 384 Pharmacol., Mol. Pharmacol. Sect. 1992, 226, 287-296.

385 (26) Fosnaric, M.; Kralj-Iglic, V.; Bohinc, K.; Iglic, A.; May, S. 386 Stabilization of pores in lipid bilayers by anisotropic inclusions. J. Phys. 387 Chem. B 2003, 107, 12519-12526.

388 (27) Mills, J. K.; Needham, D. Lysolipid incorporation in 389 dipalmitoylphosphatidylcholine bilayer membranes enhances the ion 390 permeability and drug release rates at the membrane phase transition. 391 Biochim. Biophys. Acta, Biomembr. 2005, 1716, 77-96.

392 (28) Sobko, A. A.; Kotova, E. A.; Antonenko, Y. N.; Zakharov, S. D.; 393 Cramer, W. A. Effect of lipids with different spontaneous curvature on 394 the channel activity of colicin E1: evidence in favor of a toroidal pore. 395 FEBS Lett. 2004, 576, 205-210.
(29) Leveritt, J. M., 3rd; Pino-Angeles, A.; Lazaridis, T. The structure 396 of a melittin-stabilized pore. Biophys. J. 2015, 108, 2424-2426. 397 (30) Windisch, D.; Ziegler, C.; Burck, J.; Ulrich, A. S. Structural 398 characterization of a C-terminally truncated E5 oncoprotein from 399 papillomavirus in lipid bilayers. Biol. Chem. 2014, 395, 1443-1452. 400 (31) Brogden, K. A. Antimicrobial peptides: pore formers or 401 metabolic inhibitors in bacteria? Nat. Rev. Microbiol. 2005, 3, 238-250. 402 